\title{
LONG TERM RESULTS OF DISTAL CAPITATES SHORTENING WITH CAPITOMETACARPAL ARTHRODESIS FOR TREATMENT OF EARLY STAGES OF KIENBÖCK'S DISEASE
}

By

\section{Ahmed Mahmoud Ahmed, Adnan Abd El-Aleem El-Sebaie and Galal Mansour Hegazy}

Department of Orthopedic Surgery, Faculty of Medicine, Al-Azhar University Egypt

Corresponding author Name: Ahmed Mahmoud Ahmed

Phone: +201008133441, E-mail: dr.sewafy48@ gmail.com

\begin{abstract}
Background: The aim of surgical management of Kienböck's disease has been proposed to slow the progressive osteonecrosis and secondary carpal damage.

Objective: To evaluate the effect of distal capitate shortening and arthrodesis to the base of the third metacarpal bone for treatment of the early stages of Kienböck's disease with neutral ulnar variance.

Patients and Methods: This Retrospective study was conducted on 20 patients managed at the hand surgery unit of Al-Azhar University Hospitals. Patients were divided into 2 groups by disease stage: Stage II $(n=14)$ and stage IIIA $(n=6)$. There were 13 male and 7 female, with an average age of 26.8 years. Parameters measured before and after surgery were visual analogue scale (VAS) for pain evaluation, grip strength, range of motion (ROM), ulnar variance, carpal height index, lunate height index, and the scapholunate and scaphocapitate angles. The patients were evaluated in accordance to Modified Mayo Wrist Score (MMWS).

Results: The average follow-up period was 7.13 years (range, 6.8 -7.9 years). The stage II group showed significant improvements in the mean VAS (58-5), ROM (57\% to 73\%), grip strength (54\% to $75 \%$ ), and MMWS (51-78). Patients in the stage IIIA group showed non-significant changes in mean VAS score (6442.5), ROM (52.5\% to 55.5\%), grip strength (46.5\% to57.5\%), and MMWS (36-50.5). Significant decreases in the carpal height index and scaphocapitate angle, and an increase in scapholunate angle in all stage IIIA patients were observed.
\end{abstract}

Conclusions: Distal capitate shortening with capitometacarpal arthrodesis can decrease pain and improve ROM and grip strength in patients with stage II Kienböck's disease, but not in those with stage IIIA. Also, it cannot prevent carpal collapse, especially in stage IIIA of the disease. So, this is not recommended for treating stage IIIA patients.

Keywords: Capitate shortening, distal capitate shortening, Kienböck's disease, third meta- carpal.

\section{INTRODUCTION}

Despite the advance in our understanding of Kienböck's disease and lunate vascular anatomy, the exact etiology of Kienböcke disease remain unclear, that is why its treatment is still empirical. The proper treatment is decided according to the stage of the disease at presentation (Lutsky et al., 2012).

The aim of surgical management has been proposed to slow the progression of osteonecrosis and secondary carpal 
AHMED MAHMOUD AHMED et al.,

damage or collapse. Factors defining the proposed treatment include severity of lunate damage, carpal stability, and presence of degenerative changes (Werber and Schmelz, 2012). There are different treatment options for management of Kienböck's disease (stage I,II, and IIIA) based on Lichtman classification (Lichtman et al., 2010) which aim to decompress the lunate as radial shortening, ulnar lengthening, or ulnar shortening osteotomies (Matsuhashi et al., 2011). Cadaver study has shown that radiolunate pressure decreases by $53 \%$ after partial capitate shortening (Kataoka et al., 2012).

These procedures are widely accepted for treating Kienböck's disease associated with negative or positive ulnar variance. However, these interventions can modify the distal radioulnar joint biomechanics, and lead to ulnar-sided wrist pain if used for treatment of cases with neutral ulnar variance. Van Leeuwen et al. (2016) hypothesized that radial shortening does not reverse or halt Kienböck's disease; it slows down the rate of degenerative change.

In neutral ulnar variance, capitates shortening with or without capitohamate fusion has been proposed with promising results. The aim of such procedure was of double fold benefit, i.e. lunate preservation by relieving imposed load and improving vascularization (Fouly, 2014).

However, several biomechanical studies have failed to demonstrate efficient load reduction on the lunate by adding capitate-hamate arthrodesis to capitate shortening (Gay et al., 2010).
Another technique for lunate decompression is Partial capitate shortening osteotomy, first described by Moritomo. With this procedure, good clinical and radiological results could be obtained, carpal alignment could be preserved, and better joint congruency could be provided around the scaphoid. However, mid-term/long-term results and the effects of this technique on lunate revascularization are not well known (Citlak et al., 2015).

Fouly et al. (2014) performed a distal capitate shortening with capitometacarpal arthrodesis and hypothesized that arthrodesis of shortened distal capitate to the base of third metacarpal not only reduces load to the intermediate column, but also prevents proximal migration of the capitate and progression of carpal collapse. The Camembert osteotomy, described in 2012, shortens only the radius facing the lunate, and not the scaphoid, so that the intracarpal axial compression loads are redirected toward the scaphoid (Camus et al., 2012).

The goal of our study was to evaluate the results of distal capitate shortening osteotomy with capitometacarpal arthrodesis.

\section{PATIENTS AND METHODS}

We performed a retrospective study on 20 consecutive patients ( 7 women and 13 men) who underwent distal capitate shortening osteotomy with arthrodesis to third metacarpal bone for the treatment of Kienböck's disease. All patients had stage II or IIIA disease at their initial presentation, according to the Lichtman classification system, and the diagnosis was confirmed by plain radiographs and 
magnetic resonance images (MRI) of the wrist joint. The chief symptoms were wrist pain during activity. The patients were treated between March 2012 and Aug. 2013 at Hand Surgery Unit in AlAzhar University hospitals.

The inclusion criteria were patients 18-60 years old with stage II or IIIA disease according to the Lichtman classification, with normal ulnar variance.

The exclusion criteria were patients with bilateral disease, previous surgical treatment, and less than 5 years follow-up. All enrolled patients signed written consent. Those patients were treated with distal capitate shortening and capitometacarpal arthrodesis and retrospectively reviewed (average age, $26.8 \mathrm{y}$ at the time of surgery; range, 20$33 y)$.

The preoperative and postoperative clinical parameters of all patients were evaluated with VAS and MMWS. The MMWS evaluated 4 clinical parameters: wrist pain, ROM (expressed as a percentage of the healthy side), grip strength (expressed as a percentage of the healthy side), and functional status.
Each parameter was evaluated and scored using points $(0,15,20$, or 25$)$, and the sum of points for all parameters represented the final score that was subsequently categorized into grades: excellent (90-100), good (80-90), satisfactory $(60-80)$, or poor $(<60)$. Pain analysis was performed according to the self-reported VAS score. The hand Jamar dynamometer was used to evaluate the single maximal effort of the grip strength correcting for limb dominance (Roberts et al., 2011). The total and differential arcs of motion of the wrist (extension/flexion, ulnar/radial deviation, and pronation /supination) were measured using a 2-arm Goniometer (Carter et al., 2010). The standard posteroanterior and lateral radiographic views were evaluated both before surgery and at the last follow-up. The ulnar variance was measured by perpendiculars, carpal height index according to Youm et al, lunate height index according to Stahl, and the scapholunate and the scaphocapitate angles were recorded (Fig. 1). 


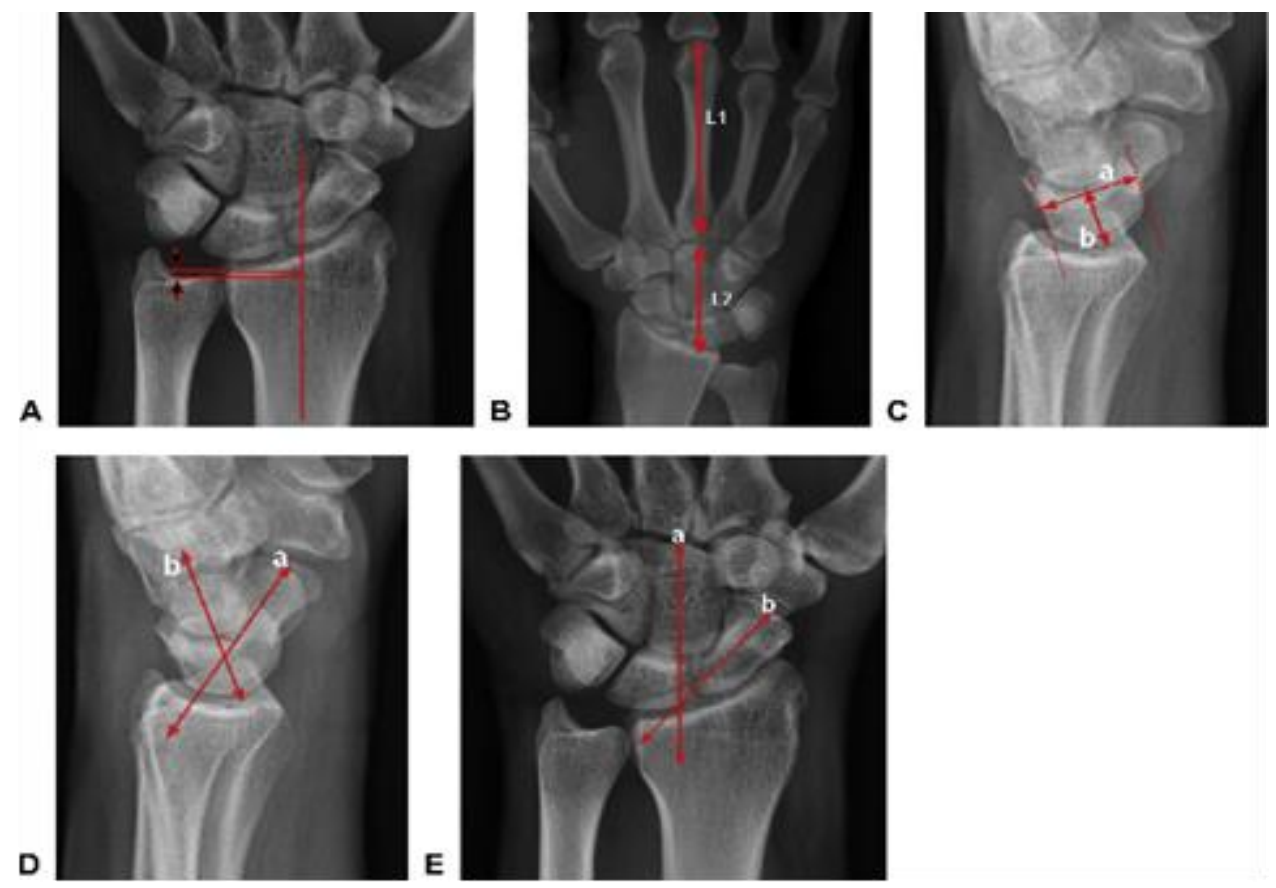

Figure (1): (A) Ulnar variance measurement (method of perpendiculars). (B) Carpal height index (method of Youm): calculated by dividing the carpal height (L2) by the length of third metacarpal (L1). (C) Lunate height index (method of Stahl): calculated by dividing the length of lunate on posteroanterior view (b) by the anteroposterior diameter of lunate on lateral view (a). (D) Scapholunate angle: it is the angle between the longitudinal axes of scaphoid (a) and lunate (b) on lateral view. (E) Scaphocapitate angle: it is the angle between the longitudinal axes of scaphoid (a) and capitate (b) on anteroposterior view.

We divided the patients into stage II group (14 patients), and stage IIIA group (6 patients). Patients were followed in the hand surgery clinic. Active finger motion was allowed immediately after surgery, and wrist joint motion was allowed 6 weeks after surgery. Active assisted and passive wrist joint motion was continued until arthrodesis occurred. Strengthening exercises were added for another 8 weeks. The postoperative radiographs were obtained immediately after surgery, after 4 weeks, every 2 weeks until capitometacarpal arthrodesis was achieved. Failure was defined as poor clinical outcomes when VAS score $>60$ mm with MMWS $<60$. Moreover, failure was considered if the disease radiographically progressed to a higher stage.

\section{Statistical Analysis:}

Data were presented as mean, standard deviations, and range. Statistical analysis was performed using paired t-tests to compare the preoperative and postoperative clinical and radiographic data. The values of the carpal height index, lunate height index, and scapholunate and scaphocapitate angles were evaluated and correlated with the clinical parameters and MMWS using the Pearson and specimen correlation with $95 \%$ confidence interval of the differences. AP value <.05 was considered statistically significant. 


\section{RESULTS}

Before surgery, the 2 groups were similar in clinical (pain, ROM, grip strength, and functional status) and radiographic parameters (ulnar variance, carpal height, and scapholunate and scaphocapitate angles) except for a significant decrease in the lunate height index in stage IIIA patients $(\mathrm{P}<0.05)$. The average follow-up period was 7.13 years (Range, $6.8-7.9$ ) years. All patients achieved bony union at the fusion site within 9-17 weeks after surgery, regardless of the plate used ( $\mathrm{Y}$ shaped /straight) or stage of the disease.
Regarding wrist pain, in the stage II group, 10 patients had complete pain relief and 4 had mild, occasional wrist pain with excessive wrist extension. Changes in parameters before to after surgery were as follows: mean VAS score 54. 29 o 2.29 ( $\mathrm{P}<0.05)$; mean ROM $52.89 \%$ to $69.29 \%$ ( $\mathrm{P}<0.05)$; and mean grip strength $44.29 \%$ to $76.43 \%$ ( $\mathrm{P}<0.05)$. The mean MMWS improved from 52.14 before surgery to 75 after surgery (P $<0.05) .10$ patients had good results and 4 had satisfactory results as per MMWS (Table 1).

Table (1): Preoperative and postoperative Clinical and Radiological parameters for stage II group

\begin{tabular}{|c|c|c|c|c|c|c|}
\hline \multirow{2}{*}{ Groups } & \multicolumn{3}{|c|}{ Preoperative } & \multicolumn{3}{c|}{ Postoperative } \\
\cline { 2 - 7 } & Range & Mean & SD & Range & Mean & SD \\
\hline VAS & $40.0-60.0$ & 54.29 & \pm 7.03 & $0.0-10.0$ & 2.29 & \pm 3.58 \\
\hline ROM (\%) & $45.0-65.0$ & 52.86 & \pm 8.25 & $60.0-80.0$ & 69.29 & \pm 7.03 \\
\hline Grip strength (\%) & $30.0-65.0$ & 44.29 & \pm 12.84 & $75.0-80.0$ & 76.43 & \pm 2.34 \\
\hline Carpal height index & $0.50-0.51$ & 0.504 & \pm 0.005 & $0.49-0.51$ & 0.497 & \pm 0.007 \\
\hline Lunate height index & $0.46-0.55$ & 0.49 & \pm 0.03 & $0.50-0.52$ & 0.51 & \pm 0.01 \\
\hline SC angle & $31.0-34.0$ & 32.43 & \pm 0.94 & $31.0-34.0$ & 32.57 & \pm 0.94 \\
\hline SL angle & $47.0-58.0$ & 52.29 & \pm 4.07 & $46.0-56.0$ & 51.29 & \pm 4.25 \\
\hline MMWS & $35.0-65.0$ & 52.14 & \pm 9.55 & $70.0-80.0$ & 75.0 & \pm 3.92 \\
\hline
\end{tabular}

(MMWS, Modified Mayo Wrist Score; ROM, range of motion; SC, scaphocapitate; SL, scapholunate; VAS, visual analog score.)

In the stage IIIA group, 4 patients had mild pain during effort that needed medication. The other 2 patients had no pain relief and did not return to work due to persistent pain and restricted joint motion 1 year after surgery. No changes were observed in VAS, ROM, grip strength, and MMWS before to after surgery. However, a significant decrease in carpal height index, lunate height index, and scaphocapitate angle and a significant increase in scapholunate angle were observed as shown in (Table 2). Concerning the Lichtman classification, 2 patients progressed to stage IIIB. Procedure failure was considered to have occurred in these patients because of persistent pain (VAS $>65 \mathrm{~mm}) 12$ months after surgery and MMWS <60. These 2 patients underwent revision surgery; one patient had proximal row carpectomy, and the other one had scaphocapitate arthrodesis. After surgery, the ROM, grip strength, and MMWS positively correlated with the postoperative carpal height index, lunate height index, and scaphocapitate angle and negatively correlated with the postoperative scapholunate angle. The VAS score negatively correlated with the postoperative carpal height index, lunate height index, and scaphocapitate angle and positively correlated with scapholunate angle (Table 3). 
AHMED MAHMOUD AHMED et al.,

Table (2): Preoperative and Postoperative Clinical and Radiographic Parameters for Stage IIIA Group

\begin{tabular}{|c|c|c|c|c|c|c|}
\hline \multirow{2}{*}{ Groups } & \multicolumn{3}{|c|}{ Preoperative } & \multicolumn{3}{c|}{ Postoperative } \\
\cline { 2 - 7 } & Range & Mean & SD & Range & Mean & SD \\
\hline VAS & $55.0-70.0$ & 65.0 & \pm 7.75 & $20.0-35.0$ & 28.33 & \pm 6.83 \\
\hline ROM (\%) & $50.0-55.0$ & 53.33 & \pm 2.58 & $55.0-70.0$ & 61.67 & \pm 6.83 \\
\hline Grip strength (\%) & $45.0-55.0$ & 48.33 & \pm 5.16 & $50.0-65.0$ & 58.33 & \pm 6.83 \\
\hline Carpal height index & $0.50-0.51$ & 0.50 & \pm 0.01 & $0.27-0.41$ & 0.34 & \pm 0.06 \\
\hline Lunate height index & $0.45-0.46$ & 0.45 & \pm 0.01 & $0.35-0.45$ & 0.40 & \pm 0.04 \\
\hline SC angle & $31.0-34.0$ & 32.67 & \pm 1.37 & $14.0-24.0$ & 19.33 & \pm 4.50 \\
\hline SL angle & $45.0-50.0$ & 48.33 & \pm 2.58 & $65.0-75.0$ & 70.0 & \pm 4.47 \\
\hline MMWS & $35.0-40.0$ & 38.33 & \pm 2.58 & $45.0-60.0$ & 53.33 & \pm 6.83 \\
\hline
\end{tabular}

Table (3): Correlations of the Postoperative Clinical Parameters $(n=20)$

\begin{tabular}{|c|c|c|c|c|c|c|c|c|}
\hline \multirow{2}{*}{ Parameters } & \multicolumn{2}{|c|}{ VAS } & \multicolumn{2}{c|}{ ROM (\%) } & \multicolumn{2}{c|}{ Grip strength (\%) } & \multicolumn{2}{c|}{ MMWS } \\
\cline { 2 - 9 } & $\mathbf{r}_{\mathbf{s}}$ & $\mathbf{p}$ & $\mathbf{r}$ & $\mathbf{p}$ & $\mathbf{r}$ & $\mathbf{P}$ & $\mathbf{r}$ & $\mathbf{P}$ \\
\hline $\begin{array}{c}\text { Carpal height } \\
\text { index }\end{array}$ & -0.583 & $0.007^{*}$ & 0.515 & $0.020^{*}$ & 0.929 & $<0.001^{*}$ & 0.909 & $<0.001^{*}$ \\
\hline $\begin{array}{c}\text { Lunate height } \\
\text { index }\end{array}$ & -0.771 & $<0.001^{*}$ & 0.343 & 0.139 & 0.722 & $<0.001^{*}$ & 0.742 & $<0.001^{*}$ \\
\hline SC angle & -0.622 & $0.003^{*}$ & 0.519 & $0.019^{*}$ & 0.936 & $<0.001^{*}$ & 0.929 & $<0.001^{*}$ \\
\hline SL angle & 0.688 & $0.001^{*}$ & -0.285 & 0.222 & -0.705 & $0.001^{*}$ & -0.812 & $<0.001^{*}$ \\
\hline
\end{tabular}

r: Pearson coefficient, rs: Spearman coefficient

\section{DISCUSSION}

In the current study, we aimed to evaluate the procedure described by Fouly et al. (2014) with regard to pain relief as measured by visual analog score (VAS), changes in wrist range of motion (ROM), grip strength, radiographic changes and success or failure as determined by clinical outcome (VAS and modified Mayo wrist score MMWS).

Mean age of included cases was 26.8 years with male predominance. In the current study, we found that there was significant decrease in VAS score postoperatively This went with Fouly et al. (2014) who reported complete pain relief in all patients. Ylldırım et al. (2020) showed that VAS pain score significantly decreased in the latest postoperative follow-up.
In the current study, we found that there was significant increase in ROM, grip strength postoperatively. Ylldirim et al. (2020) showed that wrist flexion, extension, ulnar and radial deviation values significantly improved at the latest follow-up. Although postoperative grip and pinch strength values were not completely restored compared with the healthy side, but significantly improved.

We found also significant decrease in carpal height index postoperatively while lunate height index showed insignificant differences between preoperative and postoperative. Lunate height index showed insignificantly difference between preoperative and postoperative data. While carpal height index significantly decreased in stage IIIA patients. There was also significant decrease in SC angle 
postoperatively; On the other hand, there was significant increase in SL angle postoperatively. Fouly et al. (2014) reported a significant increase in the scaphocapitate angle in all patients after surgery, and postulated that the distal capitates shortening with arthrodesis to the base of third metacarpal not only prevented carpal collapse, but also improved the carpal height.

In another previous study, biomechanical effects of capitate shortening osteotomy with capitohamate fusion (CSCHF) have been studied and the authors concluded that CSCHF increased radio scaphoid mean pressure and decreased radio lunate mean pressure and had little effect on radio carpal mean pressure. In the current study we found that there was significant increase in MMWS postoperative. In agreement with our result Fouly, et al. (2014) showed that the final postoperative modified Mayo wrist score ranged from $80-100$ points which was statistically significant from the preoperative score. Afshar (2010) showed that the mean follow-up was 12 months (range, 8-16 months). All patients demonstrated partial revascularization of the lunate and the mean revascularization time was 4.7 months (range, 3-7 months), this which was interpreted as the beginning of the revascularization process.

In patients with stage II, there were significant changes in VAS, ROM, grip strength carpal height index and MMWS postoperatively. On the other hand, there were insignificant differences between pre and postoperative regarding lunate height index, SC angle and SL angle. In the stage II, changes in parameters before to after surgery were as follows: mean VAS score 58.3 to 5 ; mean ROM $57 \%$ to $73 \%$; and grip strength $54 \%$ to $75 \%$. The mean MMWS improved from 51 before surgery to 78 after surgery. In stage II, there were significant changes in VAS, carpal height index, Lunate height index, SC angle, SL angle and MMWS postoperative. On the other hand there were insignificant differences between preoperative and postoperative regarding ROM and Grip strength. In stage IIIA group No changes were observed in VAS, ROM, grip strength, and MMWS before to after surgery. However, a significant decrease in carpal height index, lunate height index, and scaphocapitate angle and a significant increase in scapholunate angle were observed. Matholulin et al., (2010) reported combination of revascularization procedures with unloading procedures for the treatment of stage IIIA Kienböck's disease with neutral or positive ulnar variance.

\section{CONCLUSION}

The technique of distal capitates shortening with capitometacarpal arthrodesis can improve pain in patients with stage II Kienböck's disease, but cannot prevent progressive carpal collapse. It is not recommended for the treatment of stage IIIA patients.

\section{REFERENCES}

1. Afshar A, Mehdizadeh $M$ and Khalkhali $H$. (2015): Short-term clinical outcomes of radial shortening osteotomy and capitates shortening osteotomy in Kienbo "ck disease. Arch Bone Jt Surg. 3:173-178.

2. Citlak, A., Akgun, U., Bulut, T., Tahta, M., Dirim Mete, B. and Sener, M. (2015): Partial capitate shortening for Kienböck's disease. Journal of Hand Surgery (European Volume), 40(9): 957-960. 
3. Fouly, E. H., Sadek, A. F. and Amin, M. F. (2014): Distal capitate shortening with capitometacarpal fusion for management of the early stages of Kienböck's disease with neutral ulnar variance: case series. Journal of orthopaedic surgery and research, 9(1), 86.

4. Gay, A. M., Parratte, S., Glard, Y., Mutaftschiev, N. and Legre, R. (2010): Isolated capitate shortening osteotomy for the early stage of Kienböck disease with neutral ulnar variance. Plastic and reconstructive surgery, 124(2): 560-566.

5. Hugon, S., Koninckx, A. and Barbier, O. (2010): Vascularized osteochondral graft from the medial femoral trochlea: anatomical study and clinical perspectives. Surgical and radiologic anatomy, 32(9): 817-825.

6. Lichtman, D. M., Lesley, N. E and Simmons, S. P. (2010): The classification and treatment of Kienböck's disease: the state of the art and a look at the future. Journal of Hand Surgery (European Volume), 35(7): 549-554.

7. Lutsky, K., and Beredjiklian, P. K. (2012): Kienböck disease. The Journal of hand surgery, 37(9): 1942-1952.

8. Mathoulin, C. and Wahegaonkar, A. L. (2010): Revascularization of the lunate by a volar vascularized bone graft and an osteotomy of the radius in treatment of the Kienböck's disease. Microsurgery: Official Journal of the International Microsurgical Society and the European Federation of Societies for Microsurgery, 29(5): 373-378.
9. Matsuhashi, T., Iwasaki, N., Kato, H., Minami, M. and Minami, A. (2011): Clinical outcomes of excision arthroplasty for Kienbock's disease. Hand Surgery, 16(03): 277-282.

10. Roberts, H. C., Denison, H. J., Martin, H. J., Patel, H. P., Syddall, H., Cooper, C. and Sayer, A. A. (2011): A review of the measurement of grip strength in clinical and epidemiological studies: towards a standardised approach. Age and ageing, 40(4): 423-429.

11. Viljakka, T., Tallroth, K. and Vastamäki, M. (2014): Long-term outcome (20 to 33 years) of radial shortening osteotomy for Kienböck's lunatomalacia. Journal of Hand Surgery (European Volume), 39(7): 761-769.

12. Werber, K. D., Schmelz, R., Peimer, C. A., Wagenpfeil, S., Machens, H. G. and Lohmeyer, J. A. (2013): Biomechanical effect of isolated capitate shortening in Kienböck's disease: an anatomical study. Journal of Hand Surgery (European Volume), 38(5): 500-507.

13. Yıldırım, A. M., Piskin, A., Karaismailoglu, B. and Sener, M. (2020): Functional and radiological results of partial capitate shortening osteotomy in the treatment of Kienböck's disease. Journal of Hand Surgery (European Volume), 45(4): 403-407. 


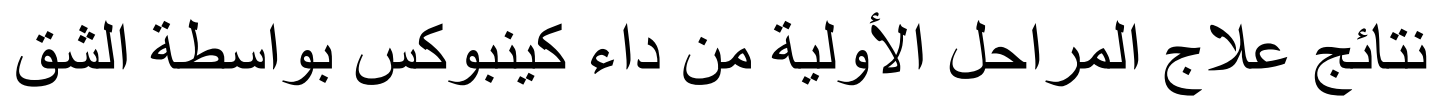

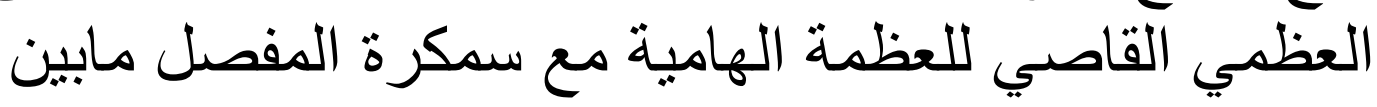
العظدة الهامية و المشطية الثالثة لليد

أحمد محمود احمد، عدنان عبدالعليم السباعي، جلال منصور حجازى

قسم جراحة العظام، كلية الطب، جامعة الأزهر

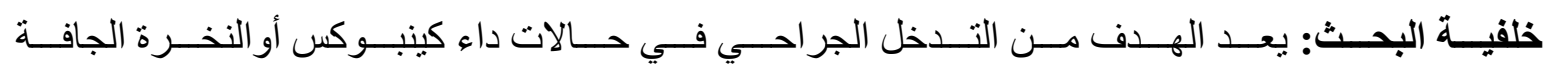

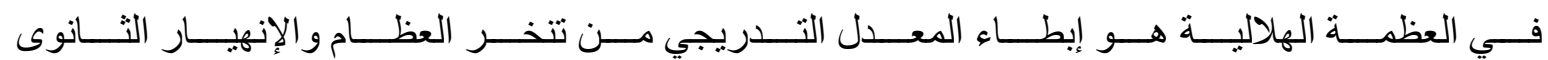

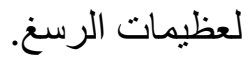

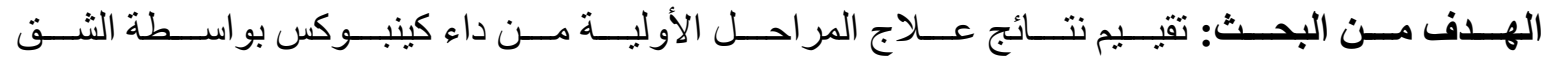

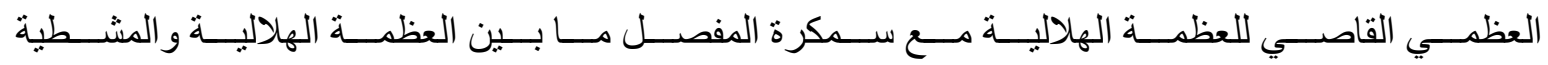
الثالثه لليد.

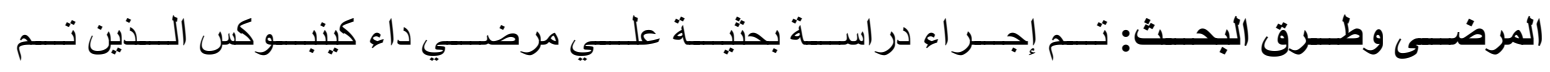

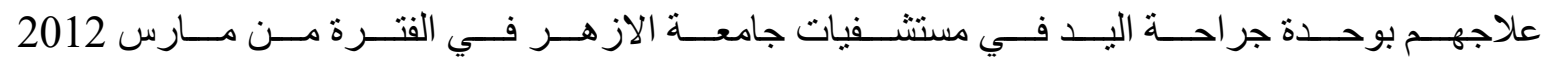

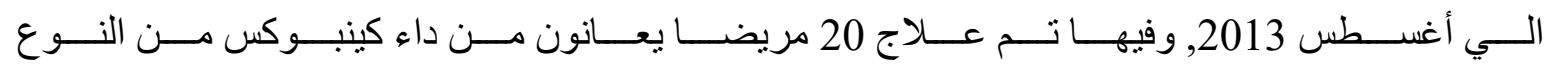

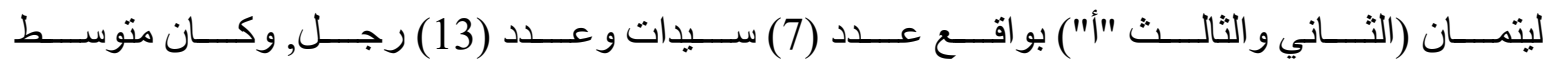

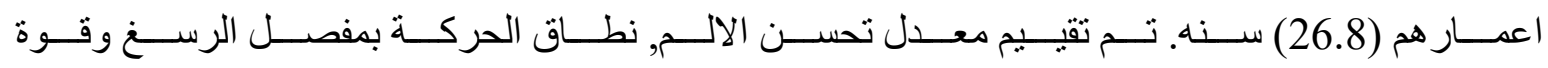
القبضة. وقد تم ضم جميع المرضى للار اسة وتحليل النتائج الوظيفية لهم.

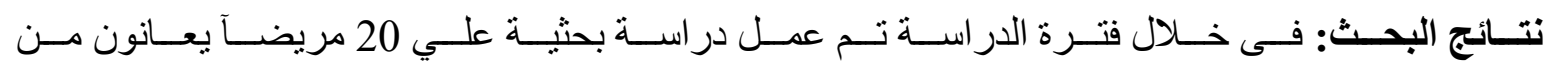

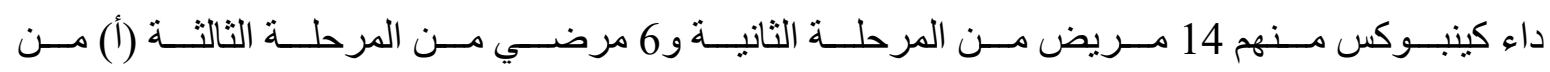

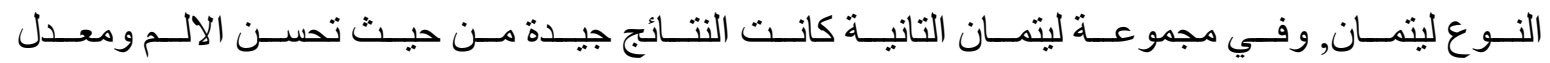
الحركة, وفي المجمو عة الثالثة (أ) كانت النتائج غير جيدة. لئنة

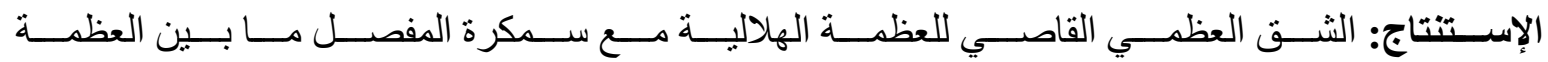

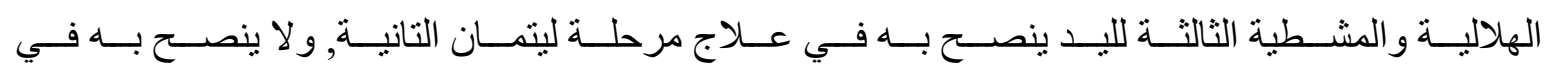

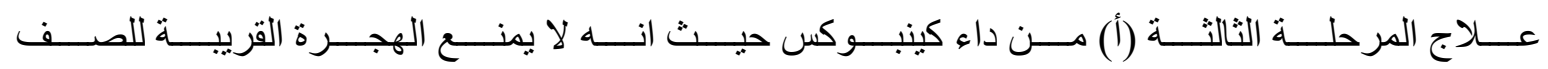

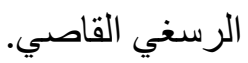

